\title{
Recent Advances in Semi-Lagrangian Modelling of Flow through the Strait of Gibraltar
}

\author{
Mohammed Seaïd $^{1}$, Mofdi El-Amrani ${ }^{2}$, and Ahmed Machmoum ${ }^{3}$
}

1 Fachbereich Mathematik AG8, TU Darmstadt, 64289 Darmstadt, Germany seaid@mathematik.tu-darmstadt.de

2 Dpto. Matemáticas, Univ. Rey Juan Carlos, 28933 Mostoles-Madrid, Spain mofdi@escet.urjc.es

3 Faculté des Sciences, LIMI, Université Ibn Zohr, B.P. 28/S Agadir, Morocco

\begin{abstract}
Two aspects of work are addressed in this paper. The first is concerned with a mathematical model for mean flow and hydraulics in the strait of Gibraltar. The model is based on the two-dimensional shallow water equations. The second aspect of work is devoted to developing a robust numerical method for solving such equations. We introduce a fully implicit semi-Lagrangian method which maintains stability even if large time steps are used in computations and reduces artificial numerical dispersion. Preliminary results obtained for a dam-break problem show that our method is able to provide stable and accurate solutions.
\end{abstract}

\section{Introduction}

During the last decade, enormous amount of work on mathematical modelling has been performed. The advent of more capable computation machines has proved the way to the use of mathematical models in all aspects of engineering, including ocean circulation, hydraulics and also floods. In this work, we try to build a numerical method to approximate solutions for the partial differential equations used to model the mean flow of water in the strait of Gibraltar and, consequently, determining their dynamics.

The strait of Gibraltar connects the Atlantic ocean with the Mediterranean sea. The differences on density, salinity and temperature of the two water bodies lead to a flow exchange through the strait. This flow exchange consists of two counter-flowing layers: (i) an upper layer of Atlantic water flowing into the Mediterranean sea and (ii) a lower layer of Mediterranean water flowing into the Atlantic ocean. For comprehensive contributions on oceanography of the strait of Gibraltar we refer the reader to the proceeding book for a seminar on studies conducted in the strait of Gibraltar 2]. In this paper, we are interested on the mean flow exchange in the water free surface to which the shallow water equations can be a suitable model. Particularly, we are concerned with numerical study of inflow contributed by the Atlantic ocean into the Mediterranean sea which takes place on the free water surface.

The main difficulties in solving numerically the shallow water equations used to model mean flow in the strait of Gibraltar are the nonlinearity structure of 
the equations and the coupling through a source function. Furthermore, it is the presence of the convective term in the shallow water equations that makes finding solutions to these equations difficult, this convective term is a source of serious computational problems and oscillations.

Eulerian methods use fixed grids and incorporate some upstream weighting in their formulations to stabilize the schemes. Among the class of Eulerian methods are the Petrov-Galerkin methods, the streamline diffusion methods and also include many other methods such as the high resolution methods from computational fluid dynamics, in particular, the Godunov methods and the essentially non-oscillatory methods. All of these Eulerian methods are easy to formulate and to implement. However, time truncation errors dominate their solutions that introduce numerical diffusion and are subject to the CFL stability conditions that put a restriction on the size of the time steps taken in numerical simulations.

Semi-Lagrangian methods, on the other hand, make use of the transport nature of the governing equations. They combine the fixed Eulerian grids with a particle tracking along the characteristic curves of the governing equations. The Lagrangian treatment in these methods greatly reduces the time truncation errors in the Eulerian methods. In addition, these methods alleviate the restrictions on the Courant number, thus allowing for large time steps in the simulations and reduce artificial numerical dispersion. Obviously, any explicit term in semi-Lagrangian formulation is computed at the characteristic feet by interpolation that might introduce extra dissipation. In order to eliminate this drawback, we propose a fully implicit semi-Lagrangian method. The discretized system can be formulated as a compact fixed point problem in flow alone. As a nonlinear solver we apply a Newton-Gmres algorithm.

The purpose of this work is to develop a robust semi-Lagrangian method to approximate solutions to the equations governing mean flow in the strait of Gibraltar. In the present paper, we discuss only the formulation of a fully implicit semi-Lagrangian method for mean flow model and results are given for a dambreak problem. As part of an ongoing project, this method will be implemented for the full model and obtained results will be compared to measurements done in the strait of Gibraltar.

\section{Mathematical Model}

In this section, we briefly set the governing equations for mean flow in the strait of Gibraltar. For more details on the modelling and related physical studies we refer to [2] and further references are cited therein. The derivation of the equations is based on Navier-Stokes problem with the assumption that the dynamics of mean flow on continental shelves is such that the vertical advection of momentum can be assumed to be small and the pressure to be approximately hydrostatic. If these hypotheses are applied to the Navier-Stokes equations, we obtain the wellknown shallow water equations in two space dimensions given by the continuity and momentum equations:

$$
\partial_{t} \eta+\partial_{x}((\eta+h) U)+\partial_{y}((\eta+h) V)=0,
$$




$$
\begin{aligned}
& \partial_{t} U+U \partial_{x} U+V \partial_{y} U-f V=-g \partial_{x} \eta-\frac{r}{\eta+h} U \sqrt{U^{2}+V^{2}}+K_{H} \nabla^{2} U, \\
& \partial_{t} V+U \partial_{x} V+V \partial_{y} V+f U=-g \partial_{y} \eta-\frac{r}{\eta+h} V \sqrt{U^{2}+V^{2}}+K_{H} \nabla^{2} U,
\end{aligned}
$$

where $\eta$ is the free surface height, $\mathbf{U}=(U, V)^{T}$ is the vertically integrated velocity, $h$ is the water depth measured from the mean sea level, $g$ is the gravity acceleration, $K_{H}$ is the horizontal eddy viscosity, $r$ denotes the drag coefficient on the bottom, $f$ is the Coriolis parameter defined by $f=2 \omega \sin \phi$, with $\omega$ is the angular velocity of the earth and $\phi$ is the geographic latitude, and $\nabla^{2}$ denotes the two-dimensional Laplace operator.

Equations (1)-(3) are defined in a spatial domain bounded by TangierBarbate line at the west and Sebta-Gibraltar at the east as shown in Fig. 1 This domain contains the Camarinal Sill (interface that separates the Mediterranean sea and the Atlantic ocean) where exchange of the water body takes place.
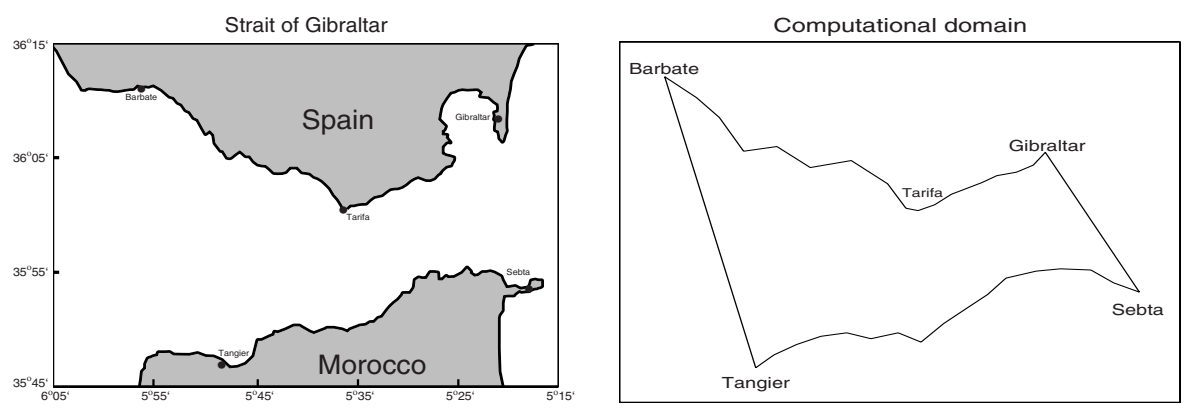

Fig. 1. Schematic diagrams for the strait of Gibraltar and the computational domain

In order to obtain a well-posed problem, initial data has to be provided and boundary conditions have to be imposed. A detailed mathematical study on these issues can be found in [2]. The overall problem is complicated one and it includes certain physical processes which are not yet fully understood. Also, the numerical methods for simulating solutions of the problem which are of key importance have not yet been well established. Theoretical considerations can provide some ideas, concerning stability, convergence rates, restriction on time stepsizes, or qualitative behavior of the solution, but a complete quantitative analysis is not possible today. Therefore, the only way to make a judgment is to perform numerical tests, at least for some problems which seem to be representative. However, looking into the literature, it seems that there have not been many studies of this type which can give satisfactory answers. 


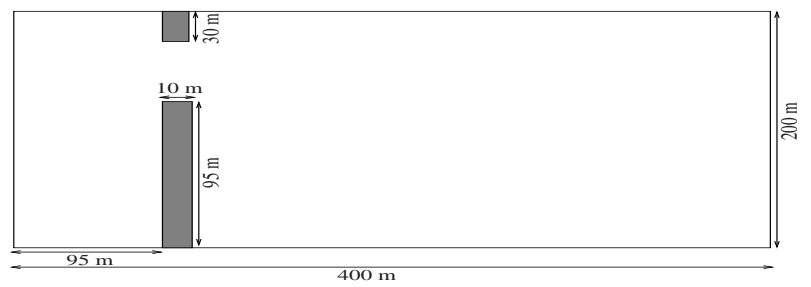

Fig. 2. Geometry of the rectangular strait in the dam-break problem

\section{Numerical Method}

The semi-Lagrangian method we consider in this paper consists on two fractional steps. The first step is the Lagrangian interpretation of the advective part in equations (11)-(3) by the modified method of characteristics, while the second step uses the Eulerian coordinates for discretization of the remaining part. In vector-valued form, the equations (10)-(13) can be rewritten as

$$
\begin{aligned}
D_{t} \eta+\mathbf{U} \cdot \nabla h+\gamma \nabla \cdot \mathbf{U} & =0, \\
D_{t} \mathbf{U}+g \nabla \cdot \eta-K_{H} \nabla^{2} \mathbf{U} & =\mathbf{S}(\gamma, \mathbf{U}),
\end{aligned}
$$

where the total depth $\gamma=\eta+h$, the source term is defined by

$$
\mathbf{S}(\gamma, \mathbf{U})=\left(f V-\frac{r}{\gamma} U \sqrt{U^{2}+V^{2}},-f U-\frac{r}{\gamma} V \sqrt{U^{2}+V^{2}}\right)^{T},
$$

the gradient vector $\nabla=\left(\partial_{x}, \partial_{y}\right)^{T}$, and $D_{t} w=\partial_{t} w+\mathbf{U} \cdot \nabla w$ is the material derivative of the function $w$ in direction of the flow $\mathbf{U}$. The fundamental idea of semi-Lagrangian methods is to impose a regular grid at the new time level, and to backtrack the flow trajectories to the previous time level. At the old time level, the quantities that are needed are evaluated by interpolation from their known values on a regular grid.

Let the time interval be divided into subintervals $\left[t_{n}, t_{n+1}\right]$ of length $\Delta t$ such that $t_{n}=n \Delta t$. Following [3], the characteristics curves of the equations (4) are the solution of initial value problem

$$
\begin{aligned}
\frac{d \mathbf{X}\left(\tau ; t_{n+1}, \mathbf{x}\right)}{d \tau} & =\mathbf{U}\left(\tau, \mathbf{X}\left(\tau ; t_{n+1}, \mathbf{x}\right)\right), \quad \tau \in\left[t_{n}, t_{n+1}\right], \\
\mathbf{X}\left(t_{n+1} ; t_{n+1}, \mathbf{x}\right) & =\mathbf{x} .
\end{aligned}
$$

Note that $\mathbf{X}\left(\tau ; t_{n+1}, \mathbf{x}\right)=\left(X\left(\tau ; t_{n+1}, \mathbf{x}\right), Y\left(\tau ; t_{n+1}, \mathbf{x}\right)\right)^{T}$ is the departure point at time $\tau$ of a fluid particle that will arrive at $\mathbf{x}=(x, y)^{T}$ at time $\tau=t_{n+1}$. The semi-Lagrangian methods do not follow the flow particles forward in time, as the Lagrangian schemes do, instead their trace backwards the position at time $t_{n}$ of particles that will reach the points of a fixed mesh at time $t_{n+1}$. By so 
doing, the semi-Lagrangian methods avoid the grid distortion difficulties that the conventional Lagrangian schemes have. In this paper, to solve the ordinary differential equations (6), we used a method first proposed in the context of semi-Lagrangian schemes to integrate the weather prediction equations [5].

Once the characteristics feet $\mathbf{X}\left(t_{n} ; t_{n+1}, \mathbf{x}\right)$ are known, the material derivatives in (4) are approximated by

$$
D_{t} \mathbf{U}=\frac{\mathbf{U}^{n+1}-\hat{\mathbf{U}}^{n}}{\Delta t}, \quad D_{t} \eta=\frac{\eta^{n+1}-\hat{\eta}^{n}}{\Delta t},
$$

where $\mathbf{U}^{n+1}=\mathbf{U}\left(t_{n+1}, \mathbf{x}\right), \eta^{n+1}=\eta\left(t_{n+1}, \mathbf{x}\right) ; \hat{\mathbf{U}}^{n}=\mathbf{U}\left(t_{n}, \mathbf{X}\left(t_{n} ; t_{n+1}, \mathbf{x}\right)\right)$ and $\hat{\eta}^{n}=\eta\left(t_{n}, \mathbf{X}\left(t_{n} ; t_{n+1}, \mathbf{x}\right)\right)$. In general, the departure points $\mathbf{X}\left(t_{n} ; t_{n+1}, \mathbf{x}\right)$ do not coincide with the spatial position of a grid point. A requirement is then that the scheme to compute $\mathbf{X}\left(t_{n} ; t_{n+1}, \mathbf{x}\right)$ be provided with a search-locate algorithm to find the host element where such point is located. A general, efficient and easy to implement scheme to perform this step in arbitrary grids is presented in [1].

Assuming that a suitable approximation is made for $\mathbf{X}\left(t_{n} ; t_{n+1}, \mathbf{x}\right)$, then the functions $\hat{\mathbf{U}}^{n}$ and $\hat{\eta}^{n}$ in (7) must be obtained by interpolation from known values at the grid points. The interpolation procedure we used in this paper is the bicubic spline most commonly used in practice.

Using the assumption that the total water depth $\gamma$ is approximately constant within the time interval $\left[t_{n}, t_{n+1}\right]$, and an implicit time integration method, the semi-discretization of (4) is

$$
\begin{aligned}
& \frac{\eta^{n+1}-\hat{\eta}^{n}}{\Delta t}+\mathbf{U}^{n+1} \cdot \nabla h+\gamma^{n} \nabla \cdot \mathbf{U}^{n+1}=0 \\
& \frac{\mathbf{U}^{n+1}-\hat{\mathbf{U}}^{n}}{\Delta t}+g \nabla \cdot \eta^{n+1}-K_{H} \nabla^{2} \mathbf{U}^{n+1}=\mathbf{S}\left(\gamma^{n}, \mathbf{U}^{n+1}\right) .
\end{aligned}
$$

Note that the bottom, $h$, is assumed to be time-independent (so there is no time-level superscript associated with it). A simple way to solve the equations (8) - (9), is to use the first equation (8) to eliminate the divergence term $\nabla \cdot \eta^{n+1}$ from the second equation (9). These procedure yields to a fixed point problem

$$
\mathbf{U}=\mathcal{H}(\mathbf{U}),
$$

in $\mathbf{U}$ only. In (10), we have dropped the $n+1$ superscript for ease of notation. Newton's method applied to (10) results in the following iteration

$$
\mathbf{U}^{(k+1)}=\mathbf{U}^{(k)}-\mathcal{R}^{\prime}\left(\mathbf{U}^{(k)}\right)^{-1} \mathcal{R}\left(\mathbf{U}^{(k)}\right),
$$

where $\mathcal{R}(\mathbf{U})=\mathbf{U}-\mathcal{H}(\mathbf{U})$ is the nonlinear residual and $\mathcal{R}^{\prime}$ is the system Jacobian approximated by a forward difference quotient of the form

$$
\mathcal{R}^{\prime}\left(\mathbf{U}^{(k)}\right) \mathbf{w} \approx \frac{\mathcal{R}\left(\mathbf{U}^{(k)}+\delta \mathbf{w}\right)-\mathcal{R}\left(\mathbf{U}^{(k)}\right)}{\delta} .
$$

If Gmres method is used to compute the Newton direction then, at each time step, the following algorithm (Newton-Gmres) has to be called in the time loop: 
$\operatorname{Algorithm~1:~} \operatorname{Ngmres}\left(\mathcal{H}, \mathbf{U}^{(0)}, \varepsilon\right)$

Given $\mathcal{H}$, tolerance $\varepsilon$ and initial guess $\mathbf{U}^{(0)}$, the Newton-Gmres algorithm for solving (10) uses the following steps: (we denote by $\operatorname{Gmres}\left(\mathbf{A}, \mathbf{b}, \mathbf{z}^{(0)}, \varepsilon\right)$ the result of Gmres algorithm applied to linear system $\mathbf{A z}=\mathbf{b}$ with initial guess $\mathbf{z}^{(0)}$ and tolerance $\varepsilon$ )

do $k=0,1, \ldots$.

Compute the residual $\quad \mathcal{R}^{(k)}=\mathbf{U}^{(k)}-\mathcal{H}\left(\mathbf{U}^{(k)}\right)$

Solve using Gmres, $\mathbf{d}^{(k)}=\operatorname{Gmres}\left(\mathcal{R}^{\prime}\left(\mathbf{U}^{(k)}\right),-\mathcal{R}\left(\mathbf{U}^{(k)}\right), \mathbf{d}^{(0)}, \varepsilon^{(k)}\right)$

Update the solution $\mathbf{U}^{(k+1)}=\mathbf{U}^{(k)}+\xi \mathbf{d}^{(k)}$

If $\left(\left\|\mathbf{U}^{(k+1)}\right\|_{L^{2}} \leq \varepsilon\right)$ stop

end do

The free surface height $\eta^{n+1}$ can be updated by back-substituting $\mathbf{U}^{n+1}$ in the first equation (8) as

$$
\eta^{n+1}=\hat{\eta}^{n}-\Delta t \mathbf{U}^{n+1} \cdot \nabla h-\gamma^{n} \Delta t \nabla \cdot \mathbf{U}^{n+1} .
$$

To discretize the equations (4) in space, we use the finite difference discretization. Thus, the spatial domain is divided into cells with nonuniform sizes and centred at grid points $\left(x_{i}=i(\Delta x)_{i}, y_{j}=j(\Delta y)_{j}\right)$. We use the notation $w_{i, j}^{n}=w\left(t_{n}, x_{i}, y_{j}\right)$ and we define the following centred difference operators

$$
\mathcal{D}_{x} w_{i, j}=\frac{w_{i+1, j}-w_{i-1, j}}{2(\Delta x)_{i}}, \quad \mathcal{D}_{x}^{2} w_{i, j}=\frac{w_{i+1, j}-2 w_{i, j}+w_{i-1, j}}{(\Delta x)_{i}{ }^{2}},
$$

similarly for $\mathcal{D}_{y} w$ and $\mathcal{D}_{y}^{2} w$. Using the semi-discrete formulation (8)-(9)), the fully-discrete approximation for the equations (4) can be directly written as

$$
\begin{aligned}
\frac{\eta_{i, j}^{n+1}-\hat{\eta}_{i, j}^{n}}{\Delta t}+ & U_{i, j}^{n+1} \mathcal{D}_{x} h_{i, j}+V_{i, j}^{n+1} \mathcal{D}_{y} h_{i, j}+\gamma_{i, j}\left(\mathcal{D}_{x} U_{i, j}^{n+1}+\mathcal{D}_{y} V_{i, j}^{n+1}\right)=0 \\
& \frac{U_{i, j}^{n+1}-\hat{U}_{i, j}^{n}}{\Delta t}+g \mathcal{D}_{x} \eta_{i, j}^{n+1}-K_{H} \tilde{\mathcal{D}}_{\mathbf{x}}^{2} U_{i, j}^{n+1}-S_{1}\left(\gamma_{i, j}^{n}, \mathbf{U}_{i, j}^{n+1}\right)=0 \\
& \frac{V_{i, j}^{n+1}-\hat{V}_{i, j}^{n}}{\Delta t}+g \mathcal{D}_{y} \eta_{i, j}^{n+1}-K_{H} \tilde{\mathcal{D}}_{\mathbf{x}}^{2} V_{i, j}^{n+1}-S_{2}\left(\gamma_{i, j}^{n}, \mathbf{U}_{i, j}^{n+1}\right)=0
\end{aligned}
$$

where $S_{1}, S_{2}$ are the entries of the source term $\mathbf{S}$ in (5) and $\tilde{\mathcal{D}}_{\mathbf{x}}^{2}=\mathcal{D}_{x}^{2}+\mathcal{D}_{y}^{2}$. The implementation of the semi-Lagrangian algorithm to solve (4) is carried out in the following steps. Given $\left\{\eta^{n}, \mathbf{U}^{n}\right\}$, we compute $\left\{\eta^{n+1}, \mathbf{U}^{n+1}\right\}$ via:

Algorithm 2: Semi-Lagrangian algorithm

Step 1. Compute the departure point $\mathbf{X}\left(t_{n+1} ; t_{n}, \mathbf{x}\right)$ in (6) and identify the element of the grid where such a point is located

Step 2. Evaluate the approximations $\hat{\eta}^{n}$ and $\hat{\mathbf{U}}^{n}$ employing the bicubic spline interpolation from the values of $\eta^{n}$ and $\mathbf{U}^{n}$, respectively

Step 3. Formulate the fixed point problem (10) for the flow $\mathbf{U}^{n+1}$ only

Step 4. Call the Newton-Gmres algorithm $\operatorname{Ngmres}\left(\mathcal{H}, \mathbf{U}^{n}, \varepsilon\right)$ to obtain $\mathbf{U}^{n+1}$

Step 5. Update the free surface height $\eta^{n+1}$ using (13)

It is worth saying that, the time integration method used in (8)-(9) is first order accurate. A second order time discretization can be formulated in similar way by considering the Crank-Nicolson method. 

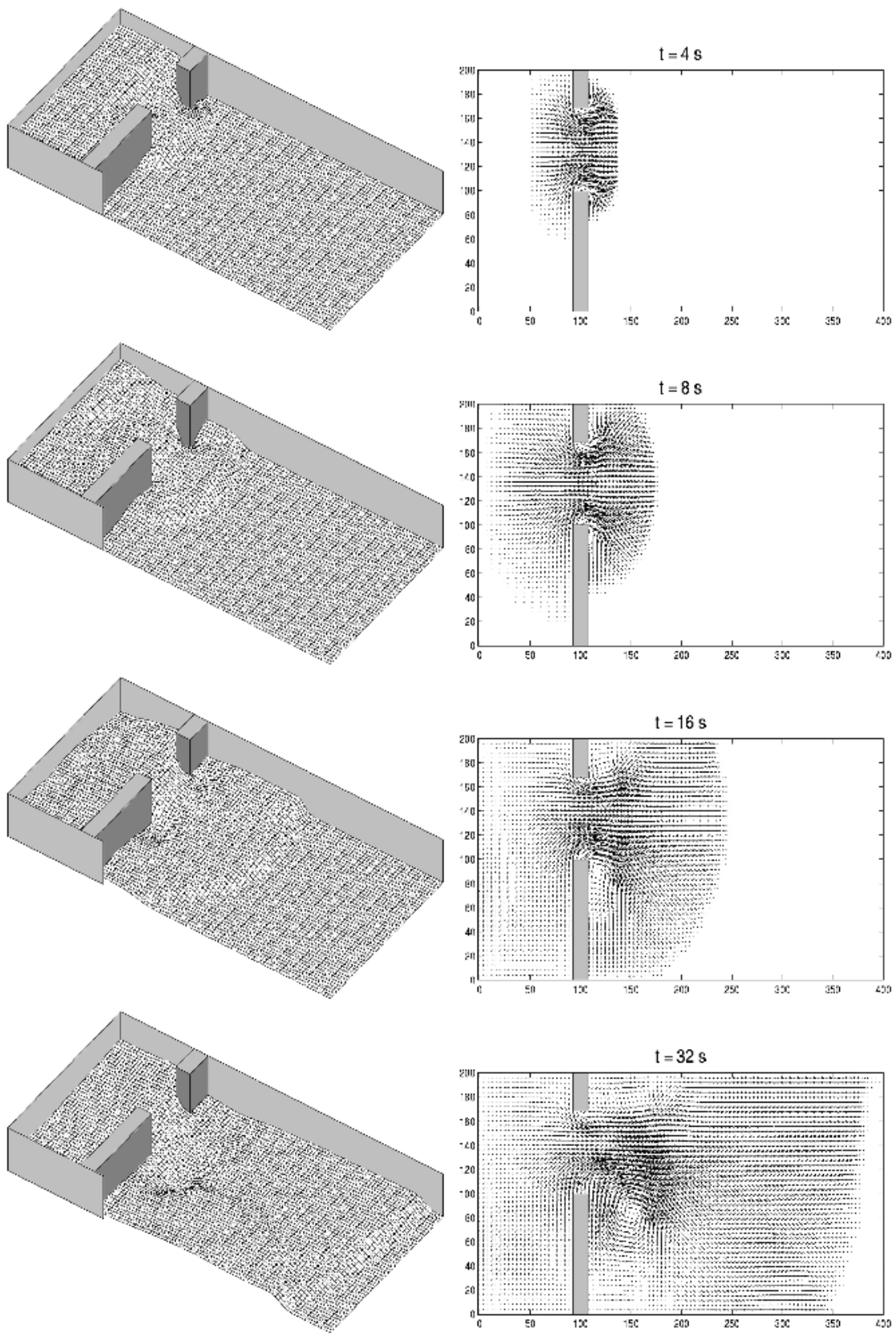

Fig. 3. Water surface elevation (left column) and velocity field (right column) 


\section{Test Problem}

To validate our algorithm we consider a prototype test of dam-break problem formulated by the equations (4). We consider a $200 \mathrm{~m}$ long and $400 \mathrm{~m}$ wide rectangular strait with two different constant levels of water separated by a dam. At $t=0 \mathrm{~s}$ part of the dam breaks instantaneously. The dam is $10 \mathrm{~m}$ thick and the breach is assumed to be $75 \mathrm{~m}$ wide. Fig. 2 shows a sketch of the strait and dimensions of the dam. The initial water level is $10 \mathrm{~m}$ at upstream and $5 \mathrm{~m}$ at downstream and water is initially at rest. For the other parameters in equations (4) we use similar values as those provided for mean flow in the strait of Gibraltar [2]. Thus, the gravity acceleration $g=9.81 \mathrm{~m} / \mathrm{s}^{2}$, the friction of the bottom $r=3 \times 10^{-3}$, the Coriolis parameter $f=8.55 \times 10^{-5} s^{-1}$, and the viscosity coefficient $K_{H}=4 \times 10^{-4} \mathrm{~kg} / \mathrm{ms}$.

In the left column of Fig. 3 we plot the water surface elevation, while the right column contains the corresponding velocity vectors. All computations are made on an uniform Cartesian grid with $\Delta x=\Delta y=40 m$ and fixed time step $\Delta t=0.5 \mathrm{~s}$. We used a tolerance $\varepsilon=10^{-5}$ to stop iterations in Newton algorithm (Algorithm 1), and we have observed that the number of iterations required to reach this tolerance was between 8 and 13 during the time integration process. The duration of simulation is $32 \mathrm{~s}$.

The semi-Lagrangian scheme captured correctly the discontinuity and the shock without need for very fine mesh. The monotonicity of the scheme is preserved and no nonphysical oscillations or extra numerical diffusion have been detected during the computations. Note that the performance of our semiLagrangian scheme is very attractive since the computed solution remains, stable, monotone and highly accurate even on coarse grids without requiring special front tracking techniques. We can see that the small complex structures of the water flow being captured by our semi-Lagrangian scheme.

\section{References}

1. Allievi, A., Bermejo, R.: A Generalized Particle Search-Locate Algorithm for Arbitrary Grids. J. Comp. Physics, 132 (1997) 157-166

2. Almazán, J.I., Bryden, H., Kinder, T., Parrilla, G. (eds.): Seminario Sobre la Oceanografía Física del Estrecho de Gibraltar. SECEG, Madrid (1988)

3. Robert, A.: A stable Numerical Integration Scheme for the Primitive Meteorological Equations. Atmos. Ocean 19 (1981) 35-46

4. Seaïd, M.: Semi-Lagrangian Integration Schemes for Viscous Incompressible Flows. J. Comp. Methods in App. Math. 4 (2002) 392-409

5. Temperton, C., Staniforth, A.: An Efficient Two-Time-Level Semi-Lagrangian SemiImplicit Integration Scheme. Quart. J. Roy. Meteor. Soc. 113 (1987) 1025-1039 